Pacific Journal of Mathematics

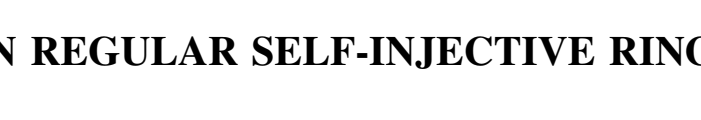




\section{ON REGULAR SELF-INJECTIVE RINGS}

\section{VAsily C. Cateforis}

If $R$ is a right non-singular ring (with 1 ) and $Q$ is its (R. E. Johnson) maximal right quotient ring, then the $R$ epimorphism $\sum_{i} p_{\imath} \otimes q_{i} \rightarrow \sum p_{i} q_{i}$ from $\left(Q \otimes_{R} Q\right)_{R}$ to $Q_{R}$ is not in general a monomorphism; in this paper we show that it is if, and only if, for each $q \in Q,(R: q)=\{r \in R \mid q r \in R\}$ contains a finitely generated large right ideal of $R$. As a corollary to this we obtain: a (Von Neumann) regular ring $R$ is right selfinjective if and only if every finitely generated nonsingular right $R$-module is projective.

All rings are associative rings with identity and all modules are unitary.

Let $R$ be a ring. If $M_{R}$ is a right $R$-module, then $Z\left(M_{R}\right)$ denotes its singular submodule [5, p. 106]. A ring $S$ containing $R$ (with same identity) is a right quotient ring of $R$ if $R_{R}$ is large [5, p.60] in $S_{R}$. If $S$ is a right quotient ring of $R$ it is easy to see that $Z\left(\left(M \otimes_{R} S\right)_{R}\right)=$ $Z\left(\left(M \otimes_{R} S\right)_{S}\right)$ and hereafter we write $Z(M \otimes S)$. Also for any left $R$-module $R^{N}$ we write $M \otimes N$ for $M \otimes_{R} N$, if no ambiguity arises.

A ring is regular (in the sense of Von Neumann) if every finitely generated right (left) ideal is generated by an idempotent [12]. For all homological notions the reader is referred to [1].

1. Rings for which $Z\left(Q \otimes_{R} Q\right)=(0)$. Let $R$ be a ring with $Z\left(R_{R}\right)=0$ and let $Q$ be its maximal right quotient $(M R Q)$ ring [5, p. 106]. It is easy to see that the mapping $\sum p_{i} \otimes q_{i} \rightarrow \sum p_{i} q_{i}$ of $\left(Q \otimes_{R} Q\right)_{R}$ onto $Q_{R}$ is an $R$-isomorphism if and only if $Z\left(Q \bigotimes_{R} Q\right)=(0)$. The latter condition does not always hold (see remarks following Theorem 2.1). In this section we obtain a characterization of those rings $R$ with $Z\left(R_{R}\right)=(0)$ for which $Z\left(Q \otimes_{R} Q\right)=(0)$.

Some definitions and consequences thereof are needed. The notion of a closed submodule is well known; we use it in the following sense:

Definition 1.1. A submodule $B_{R}$ of a right $R$-module $A_{R}$ is closed in $A$ if $B$ has no proper essential extension [5, p. 91] in $A$, i.e., if $C$ is a submodule of $A$, containing $B$ with $B$ large in $C$, then $B=C$.

Since every module has an injective essential extension in any injective module that contains it [e.g., 5, p. 92], if $A_{R}$ is injective and $B_{R}$ is closed in $A_{R}$, then $B$ is itself injective, hence a direct summand of $A$ [5, Prop. 6, p. 90].

The following lemma can be found in [9, Lemma 2.3, p. 226] and 
is stated here for easy reference:

Lemma 1.2. (a) If $B_{R}$ is a submodule of $A_{R}$ and $Z(A / B)=(0)$, then $B_{R}$ is closed in $A_{R}$.

(b) If $B_{R}$ is closed in $A_{R}$ and $Z\left(A_{R}\right)=(0)$, then $Z(A / B)=(0)$.

We recall the following definitions from [2]:

Definition 1.3. A right $R$-module $M$ is essentially finitely generated $\left(M_{R}\right.$ is EFG) if $M$ contains a finitely generated large submodule.

Definition 1.4. A right $R$-module $M$ is essentially finitely related $\left(M_{R}\right.$ is EFR) if there exists an exact sequence $0 \rightarrow K_{R} \rightarrow F_{R} \rightarrow M_{R} \rightarrow 0$ with $F_{R}$ finitely generated free and $K_{R}$ EFG.

We remark that if $M_{R}$ is EFR then and exact sequence $0 \rightarrow K_{R} \rightarrow$ $F_{R} \rightarrow M_{R} \rightarrow 0$ will have the property that $K_{R}$ is EFG whenever $F_{R}$ is finitely generated free; this follows by a result of Schanuel's contained in $[10$, p. 369].

A module $M_{R}$ is nonsingular if $Z\left(M_{R}\right)=(0)$. For brevity we write FGNS for "finitely generated nonsingular". We need the following characterization of a FGNS right $R$-module, which is EFR.

Proposition 1.5. Let $R$ be a ring with $Z\left(R_{R}\right)=(0)$ and $M R Q$ ring $Q . \quad A$ FGNS right $R$-module $M_{R}$ is EFR if and only if $Z(M \otimes Q)=(0)$.

Proof. if part. $M \otimes Q$ is $Q$-projective [9, Th. 2.7, p. 227] and hence $M_{R}$ is EFR by Proposition 1.7 [2].

only if part. $M \otimes Q$ is $Q$-projective by Proposition 1.7 [2] and hence $Z(M \otimes Q)=(0)$ since $Z(Q)=(0)$.

Now we state and prove the main result of this section:

THeOREM 1.6. Let $R$ be a ring with $Z\left(R_{R}\right)=(0)$ and $M R Q$ ring $Q$. The following statements are then equivalent:

(a) If $M_{R}$ is a FGNS right $R$-module, then $Z(M \otimes Q)=(0)$.

(b) ${ }_{R} Q$ is flat and $Z\left(Q \bigotimes_{R} Q\right)=(0)$.

(c) $(R: q)=\{x \in R \mid q x \in R\}$ is EFG for every $q \in Q$.

(d) If $B_{R}$ is a closed submodule of $F_{R}$, free of finite rank, then $B_{R}$ is EFG.

Proof. ${ }^{1}$ (a) implies (b). If $I$ is any finitely generated right

1 The author wishes to express his appreciation to the referee for indicating a cyclic proof (given here) to this theorem. 
ideal of $R$, it follows from (a) that $Z(I \otimes Q)=(0)$ and hence ${ }_{R} Q$ is flat by Theorem 1.9 [2]. Now assume $\sum_{i=1}^{n} p_{i} \otimes q_{i} \in Z\left(Q \otimes_{R} Q\right)$. If $B_{R}=p_{1} R+\cdots+p_{n} R \subseteq Q_{R}$, then the sequence $0 \rightarrow B \otimes Q \rightarrow Q \otimes_{R} Q$ is exact and $\sum p_{i} \otimes q_{i} \in Z(B \otimes Q)$. But $Z(B \otimes Q)=(0)$ from (a) so $\sum p_{i} \otimes q_{i}=0$ and $Z\left(Q \otimes_{R} Q\right)=(0)$. We have $(b)$.

(b) implies (c). Let $q \in Q$; the inclusion map $q R+R \rightarrow Q$ induces the exact sequence $0 \rightarrow(q R+R) \otimes Q \rightarrow Q \otimes_{R} Q$ by flatness of ${ }_{R} Q$ and thus $Z((q R+R) \otimes Q)=(0)$ since $Z\left(Q \otimes_{R} Q\right)=(0)$. It follows by Proposition 1.5 that $q R+R$ is EFR and hence in the exact sequence (by canonical maps) $0 \rightarrow K \rightarrow R \oplus R \rightarrow q R+R \rightarrow 0$, the module $K$ is EFG where $K=\{(r,-q r) \mid r \in(R: q)\}$. But $K \cong_{R}(R: q)$ so that $(R: q)$ is EFG for each $q \in Q$ and we have (c).

(c) implies (d). Since $B_{R}$ is closed in $F_{R}$ it follows from Lemma 1.2 (b) that $Z(F / B)=(0)$ and thus by Proposition $1.5 B$ will be EFG if $Z(F / B \otimes Q)=(0)$. Now if a module $N_{R}$ is large in a module $M_{R}$, then $Z\left(M_{R}\right)=(0)$ if and only if $Z\left(N_{R}\right)=(0)$, so it suffices to show that $F / B$ is large in $(F / B \otimes Q)_{R}$ as we may consider $F / B$ a submodule of $(F / B \otimes Q)_{R}$ [8, Prop. 2.2]. Since for any $q \in Q,(R: q)$ is large in $R_{R}$ by Proposition 1.1 (vi) [2], it follows easily from (c) and regularity of $Q$ that $(R: q) Q=Q$ for all $q \in Q$. Now let $0 \neq \sum_{i=1}^{n} x_{i} \otimes q_{i} \in F / B \otimes Q$. Since $\left(R: q_{1}\right) Q=Q$, there exists $r_{1} \in\left(R: q_{1}\right)$ such that $0 \neq\left(\sum x_{i} \otimes q_{i}\right) r_{1}$. Let $j_{1}$ be the first index $1<j_{1} \leqq n$ such that $q_{j_{1}} r_{1} \neq 0$. By the same argument there exists $r_{2} \in\left(R: q_{j_{1}} r_{1}\right)$ such that $\left(\sum x_{i} \otimes q_{i}\right)\left(r_{1} r_{2}\right) \neq 0$. Continuing the process we obtain $r_{1}, \cdots, r_{m}, m \leqq n$ elements of $R$ such that $q_{i}\left(r_{1} r_{2} \cdots r_{m}\right) \in R, i=1,2, \cdots, n$ and $\left(\sum x_{i} \otimes q_{i}\right)\left(r_{1} r_{2} \cdots r_{m}\right) \neq(0)$. We set $t=r_{1} r_{2} \cdots r_{m} \in R$ and we have $0 \neq\left(\sum x_{i} \otimes q_{i}\right) t=\sum x_{i}\left(q_{i} t\right) \otimes 1$ an element of $F / B$ in $F / B \otimes Q$. Hence $F / B$ is large in $(F / B \otimes Q)_{R}$ and thus $Z(F / B \otimes Q)=(0)$; we have $(\mathrm{d})$.

(d) implies (a). Let $0 \rightarrow K_{R} \rightarrow F_{R} M_{R} \rightarrow 0$ be an exact sequence with $F_{R}$ free of finite rank. It follows from Lemma 1.2 (a) that $K_{R}$ is closed in $F_{R}$ as $Z(F / K)=(0)$, hence EFG by (d). Thus $M_{R}$ is EFR and $Z(M \otimes Q)=(0)$ follows from Proposition 1.5.

The proof of the theorem is now complete.

2. Regular self-injective rings. We say that a ring $R$ is right self-injective if $R$ is injective as a right $R$-module. The main result in this section (Theorem 2.1) is a characterization of regular rings, which are right self-injective. In the commutative case, R.S. Pierce [7, Th.24.5, p. 106] has given a sheaf-theoretic proof of a similar characterization. We give here a ring theoretic proof of the noncommutative version.

THEOREM 2.1 For any regular ring $R$ with $M R Q$ ring $Q$ the following statements are equivalent: 
(a) $R$ is right self-injective

(b) Every FGNS right $R$-module is $R$-projective

(c) $Q_{R}$ is $R$-projective.

Proof. (a) implies (b). Let $M_{R}$ be a FGNS right $R$-module and $0 \rightarrow K_{R} \rightarrow F_{R} \rightarrow M_{R} \rightarrow 0$ an exact sequence with $F_{R}$ free of finite rank. By Lemma 1.2 (b) $K_{R}$ is closed in $F_{R}$ and since $F_{R}$ is injective the above sequence splits: $M_{R}$ is $R$-projective.

(b) implies (c). Let $M_{R}$ be any FGNS right $R$-module. From (b) and [1, Chapter I, Th. 2.2] there exists finitely generated free $R$-module $F_{R}$ such that $F_{R} \cong M_{R} \oplus B_{R}$ and hence $F \otimes Q \cong(M \otimes Q) \oplus$ $(B \otimes Q)$. Now $Z(M \otimes Q)=(0)$ follows from the fact that $Z(F \otimes Q)=$ $(0)$, as $Z(Q)=(0)$. We thus have (a) of Theorem 1.6 and hence $(R: q)$ is EFG for each $q \in Q$. Since $R$ is regular, $(R: q)=\{r \in R \mid q r \in R\}=$ $R$ for all $q \in Q$ and hence $q \in R$, all $q \in Q$ so that $R=Q$ and $Q_{R}=R_{R}$ is $R$-projective.

(c) implies (a). Since $R_{R}$ is a cyclic (finitely generated) submodule of a projective $R$-module $Q_{R}$ over a regular ring $R$, it follows by Kaplansky's Lemma $4,\left[4\right.$, p. 376] that $R_{R}$ is a direct summand of $Q_{R}$, hence $R=Q$, as $R_{R}$ is large in $Q_{R}$ and thus $R$ is right self-injective, as $Q$ is [5, Corollary, p. 107].

The proof of the theorem is now complete.

REMARKs. (1) if $R$ is any regular ring which is not right selfinjective and if $Q$ is its $M R Q$ ring, it follows from Theorem 2.1 above, that $Z\left(Q \otimes_{R} Q\right) \neq(0)$. The (weak) direct sum of infinitely many copies of a field together with the identity is such a ring with maximal (two-sided) quotient ring the direct product of the same copies of the field.

(2) The condition that $R$ be regular, in Theorem 2.1, cannot be dropped. If $R$ is a commutative integral domain with $Q$ its field of quotients, then $R=Q$ if and only if $Q_{R}$ is projective; this, however, is not the case if $R$ is an arbitrary (not regular) nonsingular ring. For example the complete ring of $2 \times 2$ matrices over a division ring is the maximal (two-sided) quotient ring of the subring of upper triangular matrices and it is right and left projective over the latter.

We next proceed to characterize those rings with singular right ideal zero over which every FGNS right module is projective. First the following lemma:

LEMMA 2.2. If $R$ is a ring with $Z\left(R_{R}\right)=(0)$ and $M R Q$ ring $Q$, then every FGNS right $R$-module $M$ can be embedded in a finitely generated free right $Q$-module $F_{Q}$. 
Proof. Set $B_{Q}=M \otimes Q$, a finitely generated right $Q$-module and let $F_{Q} \rightarrow B_{Q} \rightarrow 0$ be an exact sequence with $F_{Q}$ free of finite rank. As $F_{Q}$ is $Q$-injective (since $Q_{Q}$ is) and $Z\left(B / Z\left(B_{Q}\right)\right)=(0)$ by [3, Prop. 2.3 , p. 270] it follows that $B=Z(B) \otimes C$ where $C$ is isomorphic to a direct summand of $F_{Q}$ [9, Th.2.10, p. 119]. Now the sequence $0 \rightarrow$ $M \rightarrow M \otimes Q=B$ is exact [8, Prop. 2.2, 117] and since $M \cap Z(B)=(0)$ the sequence $0 \rightarrow M \rightarrow C$ is exact. Since $C \cong F_{Q}$ the last monomorphism is the sought embedding.

THEOREM 2.3. For a ring $R$ the following are equivalent:

(a) $Z\left(R_{R}\right)=(0)$ and every FGNS right $R$-module $M_{R}$ is projective.

(b) $R$ is right semi-hereditary, $Q_{R}$ is flat and $Z\left(Q \otimes_{R} Q\right)=(0)$.

Proof. (a) implies (b). $R$ is clearly right semi-hereditary and $Q$ is flat as a right $R$-module since it is the direct limit of its finitely generated right $R$-submodules, each one projective by (a). Finally $Z\left(Q \otimes_{R} Q\right)=(0)$ as in (b) implies (c) of Theorem 2.1.

(b) implies (a). $Z\left(R_{R}\right)=(0)$ since $R$ is right semi-hereditary. Now let $M_{R}$ be a FGNS right $R$-module. By Lemma 2.2 we may consider $M$ a submodule of some $F_{Q}$, free $Q$-module of finite rank. Since $Q_{R}$ is flat it follows that $F_{Q}$ is flat as a right $R$-module and thus $M_{R}$ is flat since submodules of flat modules are flat over a semihereditary ring (i.e., $W G D(R) \leqq 1$ ). We may conclude that $M_{R}$ is projective if we show that $M \otimes Q$ is projective as a right $Q$-module [9, Th. 2.8, p.228]. To this end consider an exact sequence $0 \rightarrow K_{R} \rightarrow$ $G_{R} \rightarrow M_{R} \rightarrow 0$ with $G_{R}$ free of finite rank. Since ${ }_{R} Q$ is also flat [2, Th. 2.1, Rks. (ii)] we obtain the exact sequence $0 \rightarrow K \otimes Q \rightarrow G \otimes Q \rightarrow$ $M \otimes Q \rightarrow 0$, which splits since $Z(M \otimes Q)=(0)$ and $G \otimes Q \cong G_{Q}$ is a $Q$-free module, which is $Q$-injective being of finite rank.

An obvious corollary of the last theorem is the well-known characterization of a Prüfer domain as an integral domain over which every finitely generated torsion-free module is projective.

The final result in this paper is a characterization of semi-simplicity of the $M R Q$ ring of a right hereditary [1] ring. First a lemma:

LEMma 2.4. Let $R$ be a ring with $Z\left(R_{R}\right)=(0)$, its $M R Q$ ring $Q$ and the property that every closed right ideal of $R$ is EFG. If $B_{Q}$ is any right ideal of $Q$, then $\left(R \cap B_{Q}\right) Q=B_{Q}$.

Proof. Obviously $(B \cap R) Q \subseteq B$. Let $q \in B$; then $q Q=e Q$ for some $e^{2}=e \in Q$ is a closed right ideal of $Q$ and hence $q Q \cap R$ is a closed right ideal of $R,[11, T h .2$, p.7] hence EFG. In particular 
$(q Q \cap R) Q=f Q, f^{2}=f \in Q$, is $Q$-injective and since $(q Q \cap R) Q \subseteq q Q$, it is a direct summand of $q Q$. But $(q Q \cap R) Q$ is a large right $R$ $(Q-)$ submodule of $q Q$, so that $(q Q \cap R) Q=q Q$. Since $q Q \cap R \subseteq B \cap R$ we have $q Q=(q Q \cap R) Q \subseteq(B \cap R) Q$ and hence $q \in(B \cap R) Q$. We have $B \cong(B \cap R) Q$.

Theorem 2.5. Suppose $R$ is a right hereditary ring and $Q$ its $M R Q$ ring. The following are equivalent:

(a) $Q$ is semi-simple.

(b) $Z\left(Q \otimes_{R} Q\right)=(0)$.

Proof. ( a ) implies ( b ). Every Q-module is $Q$-projective [1, p. 11] hence nonsingular as $Z(Q)=(0)$; we have $Z\left(Q \otimes_{R} Q\right)=(0)$.

(b) implies (a). By Remark (ii), Theorem 2.1, [2], $Z\left(R_{R}\right)=(0)$ and ${ }_{R} Q$ is flat. Now (b) gives that every closed right ideal of $R$ is $E F G$ as a special case of Theorem 1.6 (d). We next show that $Q$ is right hereditary and hence semi-simple by Osofsky's result [6]. Indeed, if $B_{Q}$ is any right ideal of $Q$ then $I=B \cap R$ is a right ideal of $R$, hence $R$-projective. Thus there exists a free $R$-module $F_{R}$ such that $F_{R} \cong I_{R} \oplus A_{R}$ and hence $F \otimes Q \cong[I \otimes Q] \oplus[A \otimes Q]$. Since $F \otimes Q$ is a $Q$-free (isomorphic to) module, it follows that $I \otimes Q$ is $Q$-projective. Since ${ }_{R} Q$ is flat, we have $I \otimes Q \cong I Q$ [5, Prop. 1, p. 132] and hence $B_{Q}$ is $Q$-projective follows from Lemma 2.2, above, as $B_{Q}=(B \cap R) Q=I Q$.

This paper is based on a portion of the author's doctoral dissertation at the University of Wisconsin. The author is deeply indebted to Professor F. L. Sandomierski, his advisor, for supplying many of the ideas included here.

\section{REFERENCES}

1. H. Cartan and S. Eilenberg. Homological algebra, Princeton University Press, Princeton, N.J.; 1956.

2. V. C. Cateforis, Flat regular quotient rings, Trans. Amer. Math. Soc. (to appear)

3. A. W. Goldie, Torsion-free modules and rings, J. of Algebra 1 (1964), 268-287.

4. I. Kaplansky, Projective modules, Ann. of Math. 68 (1958), 372-377.

5. J. Lambek, Lectures on rings and modules, Blaisdell Publishing Co., Waltham, Mass., 1966.

6. B. L. Osofsky, Rings all of whose finitely generated modules are injective, Pacific J. Math. 14 (1964), 645-650.

7. R. S. Pierce, Modules over commutative regular rings, Memoirs of the AMS, No. 70, Providence, R.I., 1967.

8. F. L. Sandomierski, Semi-simple maximal quotient rings, Trans. Amer. Math. 128 (1967), 112-120.

9. - Non-singular rings, Proc. Amer. Math. Soc. 19 (1968), 225-230.

10. R. G. Swan, Groups with periodic cohomology, Bull. Amer. Math. Soc. 65 (1959), 368-370.

11. Y. Utumi, On quotient rings, Osaka Math. J. 8 (1956), 1-18. 
12. J. Von Neumann On regular rings, Proc. Nat. Acad. Sci., U.S.A. 22 (1936), 707713.

Received April 24, 1968.

UNIVERSITY OF KENTUCKY

LeXington, KentuCky 



\section{PACIFIC JOURNAL OF MATHEMATICS}

\section{EDITORS}

H. ROYDEN

Stanford University

Stanford, California

Richard Pierce

University of Washington

Seattle, Washington 98105
J. DugundJI

Department of Mathematics

University of Southern California

Los Angeles, California 90007

BASIL GORDON

University of California

Los Angeles, California 90024

\section{ASSOCIATE EDITORS}

E. F. BECKENBACH

B. H. NeUmanN

F. WOLF

K. YOSHIDA

\section{SUPPORTING INSTITUTIONS}

UNIVERSITY OF BRITISH COLUMBIA

CALIFORNIA INSTITUTE OF TECHNOLOGY

UNIVERSITY OF CALIFORNIA

MONTANA STATE UNIVERSITY

UNIVERSITY OF NEVADA

NEW MEXICO STATE UNIVERSITY

OREGON STATE UNIVERSITY

UNIVERSITY OF OREGON

OSAKA UNIVERSITY

UNIVERSITY OF SOUTHERN CALIFORNIA
STANFORD UNIVERSITY

UNIVERSITY OF TOKYO

UNIVERSITY OF UTAH

WASHINGTON STATE UNIVERSITY

UNIVERSITY OF WASHINGTON

$* * \quad * \quad *$
AMERICAN MATHEMATICAL SOCIETY
CHEVRON RESEARCH CORPORATION
TRW SYSTEMS
NAVAL WEAPONS CENTER




\section{Pacific Journal of Mathematics}

Vol. 30, No. $1 \quad$ September, 1969

William Wells Adams, Simultaneous diophantine approximations and cubic irrationals ..................................... 1

Heinz Bauer and Herbert Stanley Bear, Jr., The part metric in convex

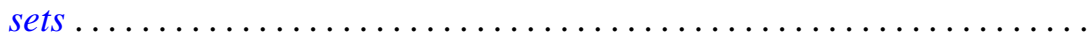

L. Carlitz, A note on exponential sums ...................... 35

Vasily Cateforis, On regular self-injective rings ................. 39

Franz Harpain and Maurice Sion, A representation theorem for measures on infinite dimensional spaces ......................... 47

Richard Earl Hodel, Sum theorems for topological spaces .............. 59

Carl Groos Jockusch, Jr. and Thomas Graham McLaughlin, Countable

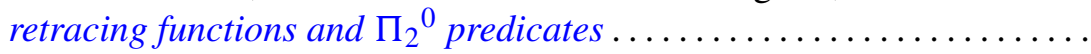

Bjarni Jónsson and George Stephen Monk, Representations of primary

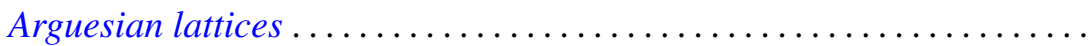

Virginia E. Walsh Knight, A continuous partial order for Peano continua...................................... 141

Kjeld Laursen, Ideal structure in generalized group algebras ........... 155

G. S. Monk, Desargues' law and the representation of primary lattices . . . 175

Hussain Sayid Nur, Singular perturbation of linear partial differential equation with constant coefficients ..........................

Richard Paul Osborne and J. L. Stern, Covering manifolds with cells ... 201

Keith Lowell Phillips and Mitchell Herbert Taibleson, Singular integrals in several variables over a local field...

James Reaves Smith, Local domains with topologically $T$-nilpotent radical....

Donald Platte Squier, Elliptic differential equations with discontinuous coefficients .................................

Tae-il Suh, Algebras formed by the Zorn vector matrix...

Earl J. Taft, Ideals in admissible algebras . .................... 259

Jun Tomiyama, On the tensor products of von Neumann algebras........ 263

David Bertram Wales, Uniqueness of the graph of a rank three group ..... 271

Charles Robert Warner and Robert James Whitley, A characterization of regular maximal ideals ......................... 